\title{
Regulatory actions of ToxR and CalR on their own genes and type III secretion system 1 in Vibrio parahaemolyticus
}

\author{
George Osei-Adjei ${ }^{1, *}$, He Gao ${ }^{3, *}$, Ying Zhang ${ }^{1}$, Lingyu Zhang ${ }^{1}$, Wenhui Yang ${ }^{2}$, \\ Huiying Yang ${ }^{2}$, Zhe Yin ${ }^{2}$, Xinxiang Huang ${ }^{1}$, Yiquan Zhang ${ }^{1}$ and Dongsheng Zhou ${ }^{2}$ \\ ${ }^{1}$ School of Medicine, Jiangsu University, Zhenjiang 212013, China \\ ${ }^{2}$ State Key Laboratory of Pathogen and Biosecurity, Beijing Institute of Microbiology and Epidemiology, Beijing 100071, China \\ ${ }^{3}$ State Key Laboratory of Infectious Disease Prevention and Control, National Institute for Communicable Disease Control \\ and Prevention, Chinese Center for Disease Control and Prevention, Beijing 102206, China \\ *These authors have contributed equally to this work \\ Correspondence to: Dongsheng Zhou, email: dongshengzhou1977@gmail.com \\ Yiquan Zhang, email: zhangyiquanq@163.com \\ Xinxiang Huang, email: huxinx@ujs.edu.cn \\ Keywords: Vibrio parahaemolyticus, calR, ToxR, T3SS 1 \\ Received: May 10, $2017 \quad$ Accepted: June 20, $2017 \quad$ Published: July 22, 2017 \\ Copyright: Osei-Adjei et al. This is an open-access article distributed under the terms of the Creative Commons Attribution License \\ 3.0 (CC BY 3.0), which permits unrestricted use, distribution, and reproduction in any medium, provided the original author and \\ source are credited.
}

\section{ABSTRACT}

Vibrio parahaemolyticus is the leading cause of seafood-associated gastroenteritis. Type III secretion system 1 (T3SS1) is one of the virulence determinants of this bacteria. T3SS1 expression is regulated by ToxR and CaIR. ToxR represses the transcription of T3SS1 genes via activation of CaIR, which acts as a transcriptional repressor of T3SS1 genes. However, the transcriptional regulation mechanisms have not been elucidated. As showing in the present work, ToxR binds to the promoter DNA region of calR to activate its transcription. CalR occupies the promoter-proximal regions of each detected target operons in T3SS1 loci to repress their transcription, and thereby inhibiting T3SS1-dependent cytotoxicity. Moreover, a feedback CalR inhibits toxR and its own gene in a direct manner. Collectively, this work reported an interesting gene regulatory network involving the reciprocal regulation of ToxR and CaIR, and their regulation on T3SS1 genes transcription in $V$. parahaemolyticus.

\section{INTRODUCTION}

Vibrio parahaemolyticus, the etiological agent of gastroenteritis, is a Gram-negative halophilic bacterium mostly found in marine and estuarine habitats. Human infections occurs through the consumption of contaminated poorly cooked or raw seafood with clinical manifestations like diarrhea, nausea, vomiting and abdominal cramping [1]. V. parahaemolyticus strains can also cause skin infection and septicemia if the bacterium enters into open wounds [1,2]. V. parahaemolyticus has been recognized as a leading cause of seafood associated gastroenteritis [3].

The type III secretion system (T3SS), a novel needlelike bacterial protein injection machinery, is used by most
Gram-negative bacteria to deliver toxic proteins called effectors into host eukaryotic cytoplasm, where they can manipulate host cell function [4]. The V. parahaemolyticus strain RIMD2210633 possesses two T3SS loci, named as T3SS1 (vp1656-1702) and T3SS2 (vpa1321-1731), respectively [5]. T3SS1 predominantly contributes to $V$. parahaemolyticus-induced cytotoxicity in most mammalian cell lines and lethality in the mouse infection model in vivo [6, 7]. T3SS1 destroy host cells through autophagy, membrane blebbing, cell-rounding and cell lysis $[8,9]$. T3SS2 is related to $V$. parahaemolyticus enterotoxicity with symptoms such as diarrhea, disruption of the intestinal epithelia and inflammation [6]. In the recent years, many toxic effectors of T3SS1 and 2 that are involved in pathogenesis were identified in V. parahaemolyticus [7, 10-15]. 
ToxR is a transmembrane regulatory protein that functions in DNA binding and transcriptional regulation in pathogenic Vibrio species. In $V$. cholerae, disease occurs due to the role of ToxR which acts with TcpP to induce toxT expression [16, 17]. ToxT in turn induces expression of colonization factors and cholera toxin [17]. ToxR also can directly activate the cholera toxin genes $\operatorname{ctx} A B$ expression in the presence of bile acids, as well as some outer membrane proteins, ompT and ompU [18-20]. Kazi et al., in their recent work demonstrated that ToxR shares more than a third of its regulon with the histone-like nucleoid structuring protein $\mathrm{H}-\mathrm{NS}$, and antagonizes H-NS for control of critical colonization functions in $V$. cholerae [21]. ToxR in $V$. parahaemolyticus is well conserved with that of in $V$. cholerae [22]. Previous studies demonstrated that ToxR induces the expression of TDH (hemolytic activity), T3SS2 (enterotoxicity), and OmpU (fitness), but the molecular mechanisms have not been fully elucidated [22-24]. ToxR also represses the transcription of T3SS1 genes via activation of CalR, which acts as a repressor of T3SS1 genes [23].

$V$. parahaemolyticus CalR, a LysR-type transcriptional regulator, was originally described as a transcriptional repressor of swarming motility and T3SS1 expression [25]. Our recent study reported of a new physiological role for CalR as a repressor of the tdh2 transcription, and thereby inhibiting hemolytic activity against human type $\mathrm{O}$ erythrocytes [26]. We also demonstrated that CalR is a positive regulator of the adhesion of $V$. parahaemolyticus to HeLa cells through direct acting on the three putative operons of T6SS2 locus, as well as a transcriptional activator of $c p s Q-m f p A B C$ and $m f p A B C$ in a direct and indirect manner, respectively [27, 28]. Expression of CalR itself is induced by cold shock, low concentration of sodium and calcium ions, and the regulatory protein ToxR [25, 29, 30].

In the present work, we present an interesting gene regulatory network involving the reciprocal regulation of ToxR and CalR, and negative regulation of T3SS1 and calR by CalR in $V$. parahaemolyticus (Figure 1). We report that ToxR has a direct and positive regulation on calR transcription, while CalR inhibits T3SS1 genes expression in a direct manner, and thus ToxR represses T3SS1 genes transcription via direct activation of CalR. As a feedback loop, CalR inhibits tox $R$ and its own gene calR in a direct manner. Thus, the transcription of T3SS1 genes is tightly regulated by ToxR and CalR in $V$. parahaemolyticus.

\section{RESULTS}

\section{Positive regulation of calR by ToxR}

The qRT-PCR assay was conducted to detect the mRNA levels of the target genes in WT and $\Delta$ toxR (Figure 2A). The result indicated that the mRNA level of calR was greatly decreased in $\Delta$ tox $R$ relative to $\mathrm{WT}$. The primer extension assay (Figure 2B) further disclosed that the mRNA level of calR decreased in $\Delta$ tox $R$ relative to WT. The recombinant lacZ fusion plasmid that contains the indicated promoter-proximal region and promoterless lacZ gene was transformed into WT and $\Delta$ toxR, respectively, to test the action of ToxR on the promoter activity of calR. The results showed that the calR promoter activity significantly decreased in $\Delta$ tox $R$ relative to WT (Figure 2C). The entire promoter DNA regions of calR was amplified, purified, and subjected to EMSA with purified His-ToxR protein (Figure 2D). The result showed that His-ToxR was able to bind to the upstream DNA

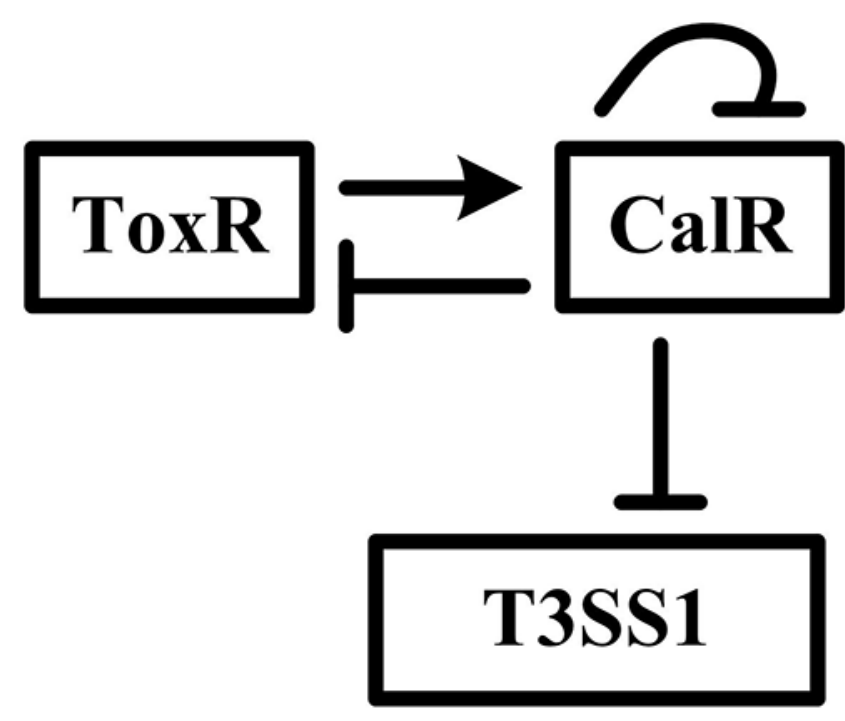

Figure 1: Gene regulatory circuit. The details for regulatory circuit are described in the main text. The arrow line represents positive regulation. The vertical lines represents negative regulation. 
fragment of calR promoter in a dose dependent manner. As further determined by DNase I footprinting (Figure 2E), His-ToxR protected a single DNA region from 286 to 257 bp upstream of calR against DNase I digestion considered as the ToxR site. Taken together, ToxR activates calR transcription in a direct manner.

\section{Negative regulation of toxR by CalR}

The primer extension assay detected a single transcription start site at $101 \mathrm{bp}$ upstream of toxR, and its transcriptional activity is under the negative control of
CalR (Figure 3B). The qRT-PCR and LacZ fusion assays further confirmed the negative correlation between CalR and toxR transcription (Figure 3A, and 3C). EMSA results showed that His-CalR was able to bind to the upstream DNA fragment of tox $R$ in a dose dependent manner and the His-CalR proteins at all amounts used could not bind to the $16 \mathrm{~S}$ rDNA fragment as the negative control (Figure 3D). As further determined by DNase I footprinting (Figure 3E), His-CalR protected two different DNA regions upstream of toxR against DNase I digestion that were considered as the CalR sites. Taken together, CalR represses the transcription of tox $R$ in a direct manner.

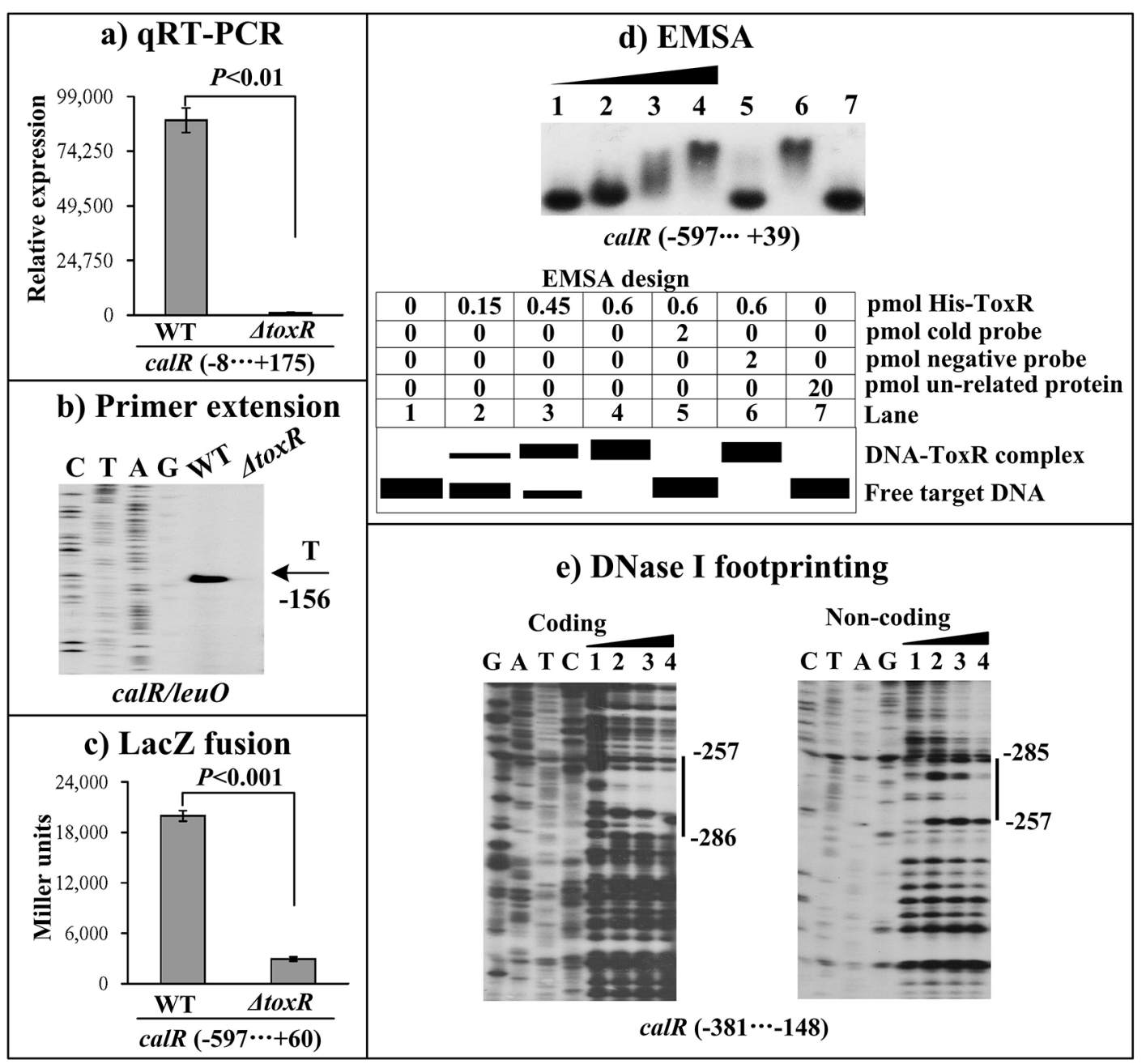

Figure 2: ToxR activates the transcription of calR. (A) qRT-PCR. The relative mRNA level of calR was compared between $\triangle t o x R$ and WT. (B) Primer extension. An oligonucleotide primer was designed to be complementary to the RNA transcript of calR. The primer extension products were analyzed with an $8 \mathrm{M}$ urea $-6 \%$ acrylamide sequencing gel. The transcription start site is indicated by the arrow with nucleotide and position. (C) LacZ fusion assay. The entire promoter-proximal region of toxR was cloned into pHRP309, and then transformed into WT or $\Delta$ toxR to determine the $\beta$-galactosidase activity (miller units) in cellular extracts. (D) EMSA. The entire promoter-proximal region of calR was incubated with increasing amounts of purified His-ToxR protein, and then subjected to $6 \%$ (w/v) polyacrylamide gel electrophoresis. Shown below the binding is the schematic representation of the EMSA design. (E) DNase I footprinting. Labeled coding or non-coding DNA probes were incubated with increasing amounts of purified His-ToxR (Lanes 1, 2, 3, and 4 containing $0,0.2,0.6$, and $0.8 \mathrm{pmol}$, respectively), and subjected to DNase I footprinting assay. The footprint regions were indicated with vertical bars. The negative and positive numbers represent the nucleotide position upstream and downstream of calR, respectively. Lanes C, T, A and G represent the Sanger sequencing reactions. 


\section{Autoregulation of CalR}

The primer extension assay detected a single transcription start site at $156 \mathrm{bp}$ upstream of calR which was under the negative control of CalR (Figure 4A). The lac $Z$ fusion result showed that the promoter activity of calR is significantly enhanced in $\Delta c a l R$ than that in WT (Figure 4B), suggesting a negative correlation between CalR and its own gene transcription. The EMSA and DNase I footprinting assays were conducted to investigate the binding activity of CalR to its own promoter DNA.
The results showed that His-CalR protects a single DNA region from $121 \mathrm{bp}$ to $182 \mathrm{bp}$ upstream of calR against DNase I digestion in a dose dependent manner (Figure 4C, and 4D). Thus, these results indicated the direct binding activity and the negative autoregulation of CalR.

\section{CalR represses the cytotoxic activity against HeLa cells}

V. parahaemolyticus cytotoxic activity against the HeLa cells was evaluated in terms of the release of LDH

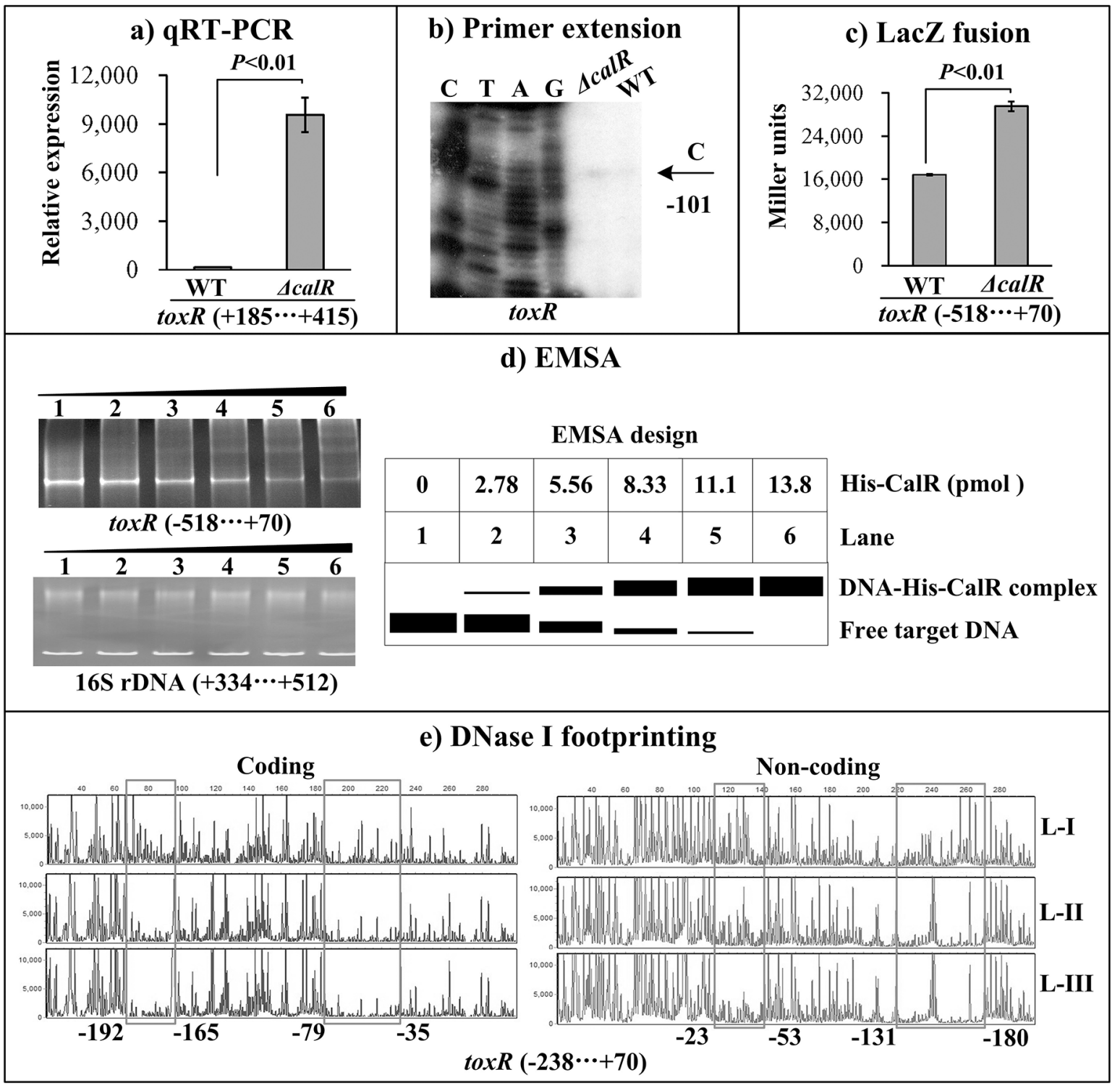

Figure 3: CalR represses the transcription of toxR. The negative and positive numbers represent the nucleotide position upstream and downstream of toxR, respectively. (A) qRT-PCR, (B) primer extension, and (C) LacZ fusion were done as Figure 2. (D) EMSA. The promoter DNA region of tox $R$ was incubated with increasing amounts of purified His-CalR, and then subjected to $6 \%$ (w/v) polyacrylamide gel electrophoresis. The DNA bands were visualized by EB staining. Shown below the EMSA results is the EMSA design. (E) DNase I footprinting. The promoter fragment of toxR was labelled with FAM or HEX, incubated with increasing amounts of purified His-CalR (Lanes-I, II, and III containing 0, 5.52, and 11.04 pmol, respectively), and then subjected to DNase I footprinting assay. The fragments length was analyzed using an ABI 3500XL DNA analyzer. The footprint regions were boxed and marked with positions. 


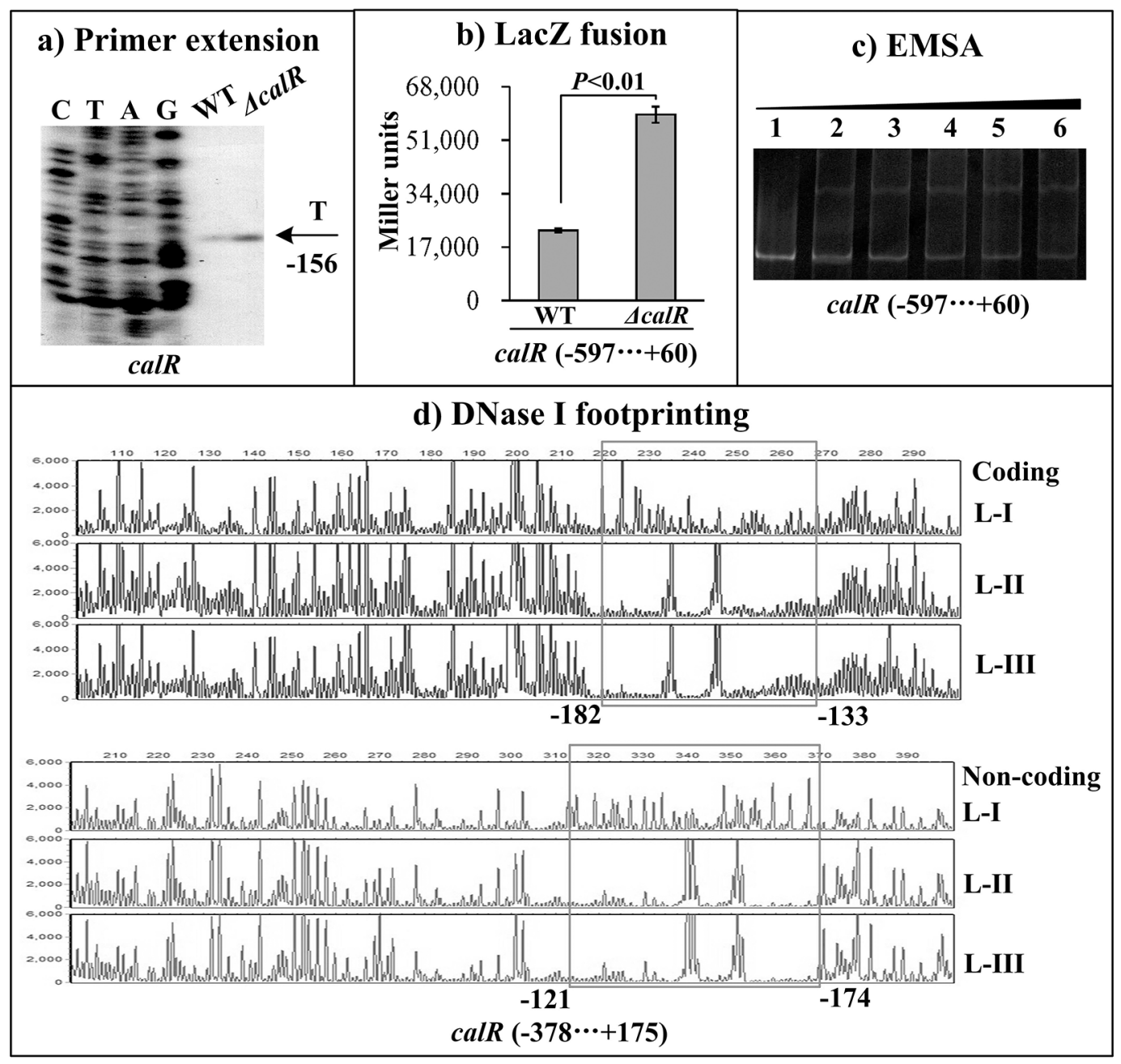

Figure 4: Autoregulation of $\mathbf{c a l R}$. The negative and positive numbers represent the nucleotide position upstream and downstream of calR, respectively. (A) qRT-PCR, (B) primer extension, and (C) LacZ fusion were done as Figure 2. (D) EMSA and (E) DNase I footprinting were done as Figure 3.

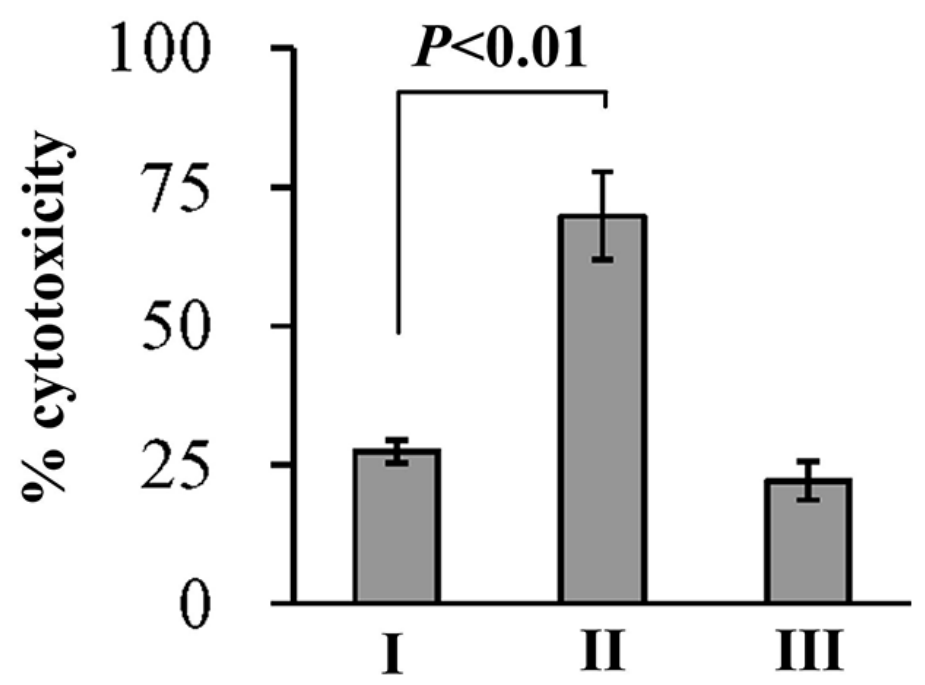

Figure 5: Cytotoxicity against HeLa cells of $\boldsymbol{V}$. parahaemolyticus strains. HeLa cells were infected with $V$. parahaemolyticus strains at $[\mathrm{MOI}]=1: 2.5$. The percentage cytotoxicity was calculated as HeLa cells killed/input bacterial cells. I, II, and III represent WT/ pBDA33, $\Delta$ calR/pBDA33 and $\Delta c a l R / p B D A 33-c a l R$, respectively. 
from cultured cells (Figure 5). The cytotoxicity against the HeLa cells infected with $\triangle c a l R /$ pBDA33 was significantly increased than that of $\mathrm{WT} / \mathrm{pBDA} 33$ or $\mathrm{c}-\Delta$ calR, while there was no significant change between $\Delta c a l R / \mathrm{pBAD} 33$ calR and WT/pBAD33. These results confirmed that $V$. parahaemolyticus CalR acts as a repressor of the cytotoxic activity against HeLa cells.

\section{Negative regulation of T3SS1 genes by CalR}

T3SS1 locus is composed of at least ten putative operons. The three operons vp1700-1688 (exsBAD$v s c B C D), v p 1667-1655$ and $v p 1687-1686$ were arbitrarily selected, and the first genes of each of the operons were subjected to the following investigations: qRT-PCR, primer extension, LacZ fusion, EMSA and DNase I footprinting. The qRT-PCR results indicated that the mRNA level of each target gene was greatly increased in $\triangle$ calR relative to WT (Figure 6A). The primer extension assay disclosed a single transcription start site for each of the three operons, and their transcription activities were under the negative control of CalR (Figure 6B). The lacZ fusion results showed that the promoter activity of each of the three operons in $\Delta c a l R$ was much higher relative to that in WT (Figure 6C). EMSA results showed that His-CalR was able to bind to each of the target DNA fragment in a dose-dependent manner (Figure 6D). As further determined by DNase I footprinting (Figure 6E), His-CalR protected two different DNA regions upstream of vp1667 against DNase I digestion that were considered as the CalR sites; however, a single DNA region upstream was protected against DNase digestion in both exs $B$ and $v p 1687$. The above results revealed the negative regulation of each target gene by CalR, respectively.

\section{Promoter structure of target genes}

The primer extension assays detected a single transcriptional start site for each target gene, through which we determined the corresponding -10 and -35 elements. The DNase I footprinting assays identified one or more footprint sites for each target promoter-proximal region, which is considered as the ToxR or CalR binding sites. Thus, we depicted the structural organization of the promoter of toxR, calR, exsB, vp1687 and $v p 1667$ by collecting the data of the translation/transcription start sites, promoter -10 and -35 elements, CalR sites, and Shine-Dalgarno (SD) sequences (ribosomal binding sites) (Figure 7).

\section{DISCUSSION}

T3SSs are tightly regulated protein secretion systems that are used by most Gram-negative bacteria to deliver toxic effectors into host eukaryotic cytoplasm [4]. T3SS gene expression is sophisticatedly regulated by the transcriptional regulatory system ExsACDE. In Pseudomonas aeruginosa, ExsA is a positive regulator of T3SS expression [31]. ExsD acts as an anti-activator by complexing with ExsA, which prevents ExsA fuction [31]. ExsC is an anti-anti-activator that binds ExsD to prevent the ExsD-ExsA complex formation, thereby

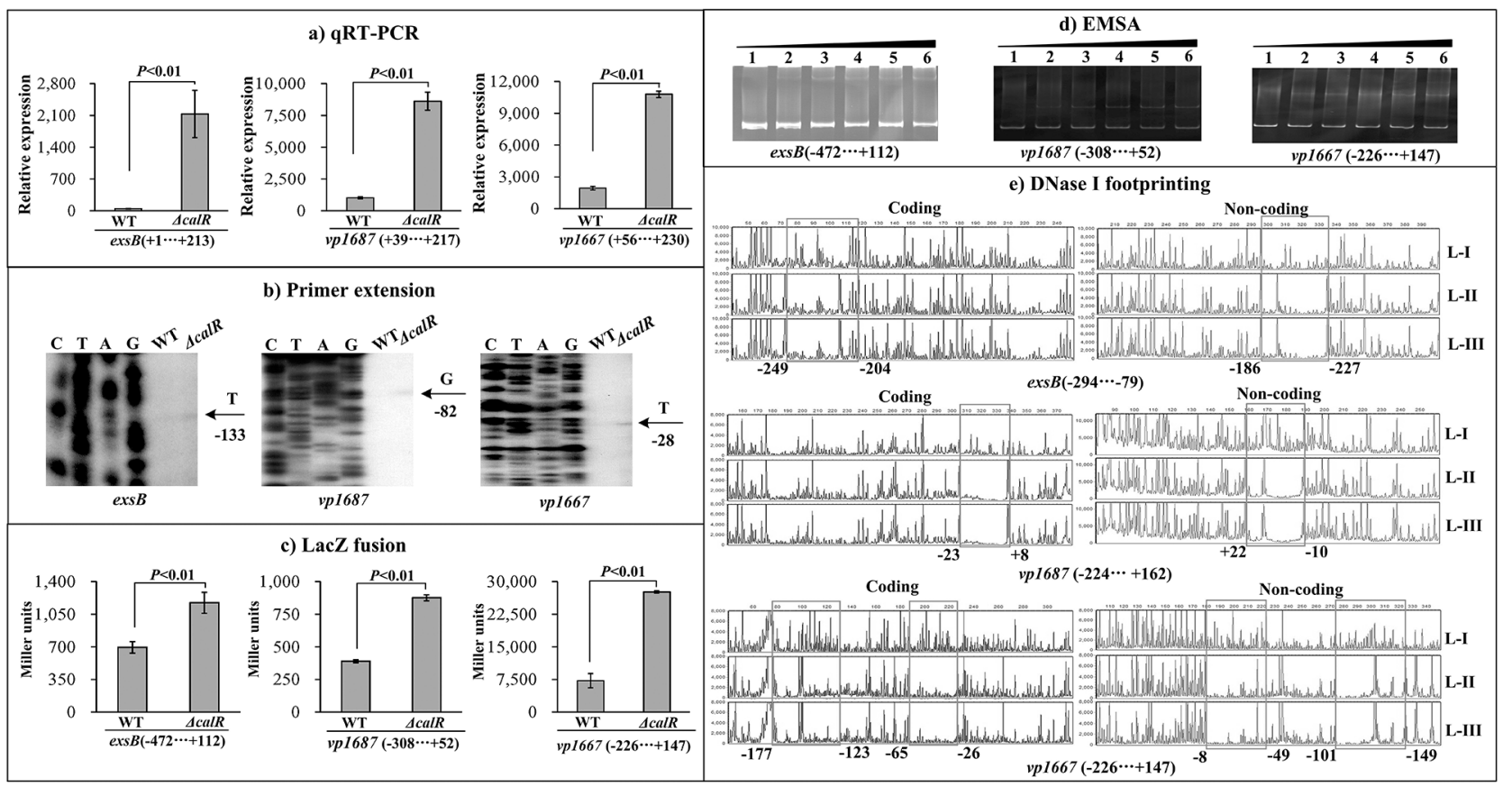

Figure 6: CalR represses the transcription of T3SS1 genes. (A) qRT-PCR, (B) primer extension, and (C) LacZ fusion were done as Figure 2. (D) EMSA and (E) DNase I footprinting were done as Figure 3. 
allowing ExsA to activate T3SS gene expression [31]. ExsE is a secreted substrate of the T3SS that binds ExsC and antagonizes the regulatory activity of ExsC [31]. The expression of T3SS1 genes in $V$. parahaemolyticus is similar to the ExsACDE regulatory cascade in $P$. aeruginosa [32, 33]. Sun and his colleagues previously reported that $\mathrm{H}-\mathrm{NS}$ binds to the promoter regions of $\operatorname{exs} A$, $\operatorname{exs} C$, and $\operatorname{exs} D$ to repress their transcription, and thereby inhibiting T3SS1 expression and $V$. parahaemolyticus- induced cytotoxicity [34, 35]. High level of T3SS1 expression occurs by growing bacteria in high-calcium or low-iron growth conditions [25]. LafK, a key $\sigma^{54}$ dependent regulator, appears to be required to mediate the major portion of the T3SS1 response to high-calcium [25]. By contrast, CalR, which negatively regulates T3SS1 transcription, is required for the low calcium-mediated decrease of T3SS1 gene expression [25]. Although the regulatory mechanisms were not clarified, Whitaker et al.,

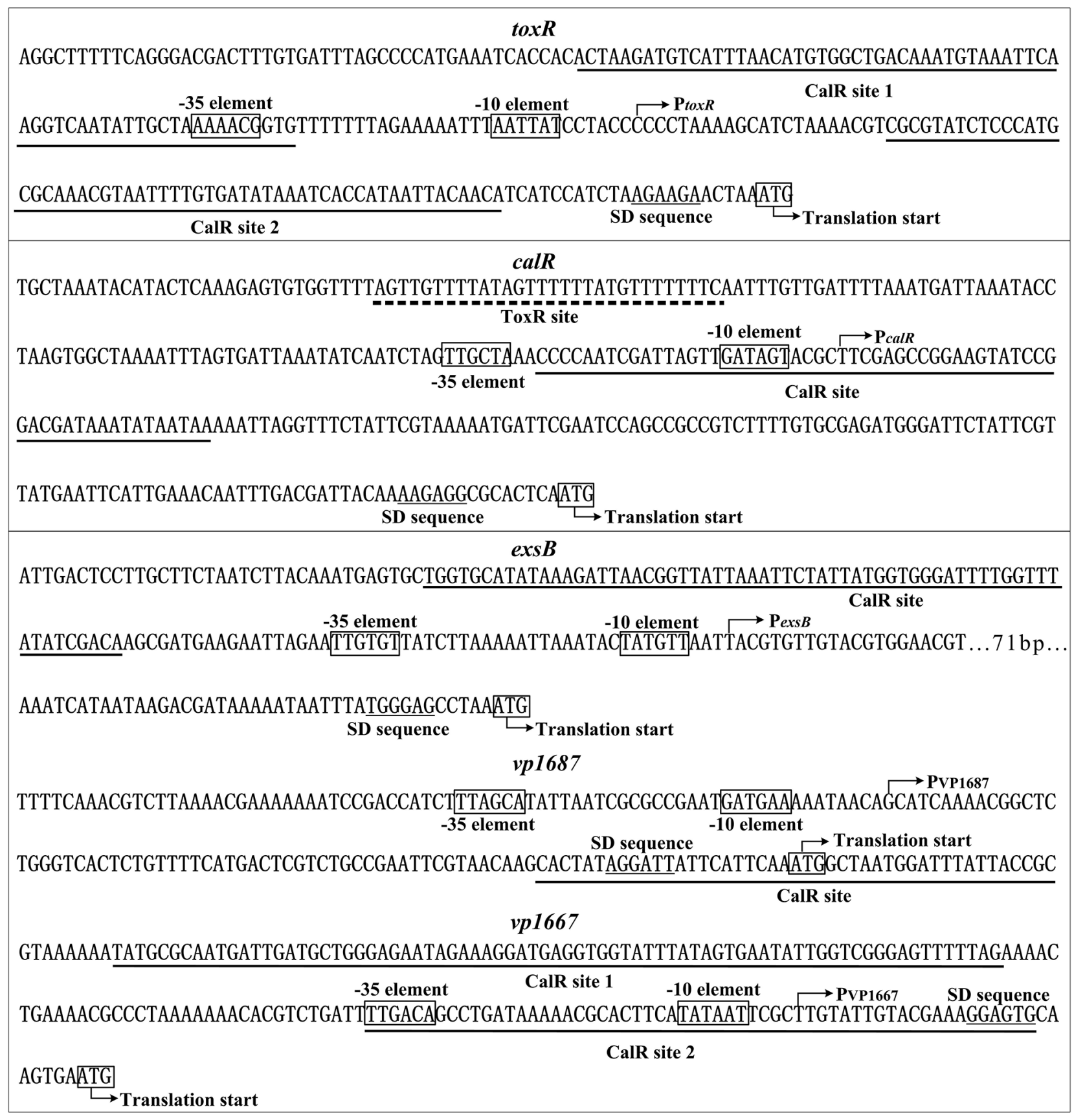

Figure 7: Structural organization of target promote rs. The DNA sequence was derived from RIMD 221063. The transcription start site is indicated by bent arrows. The SD box and -10/-35 elements are enclosed in boxes. The CalR sites are underlined with solid lines, while the ToxR site is underlined with a broken line. 
Table 1: Oligonucleotide primers used in this study

\begin{tabular}{|c|c|}
\hline Type of analysis and primer & Sequences $\left(5^{\prime}-3^{\prime}\right)$ \\
\hline \multicolumn{2}{|l|}{ Construction of mutants } \\
\hline calR-A & GTAGCTGCAGGCAGATTATTTGACTGATACGC \\
\hline calR-B & GTTCGCAAATGGGAAGTCTCTCATCGCATCTTTCTTCTC \\
\hline calR-C & GAGAAGAAAGATGCGATGAGAGACTTCCCATTTGCGAAC \\
\hline calR-D & GTGAGCATGCTACTTACCTTTTGGCTTACAG \\
\hline toxR-A & GTGACTGCAGAAACGCAATTTGTCTGATG \\
\hline tox $R-\mathrm{B}$ & ATCTTCATGCTGGCCTCCTTTAGTTCTTCTTAGATGGATGATG \\
\hline toxR-C & CATCATCCATCTAAGAAGAACTAAAGGAGGCCAGCATGAAGAT \\
\hline toxR-D & GTGAGCATGCAATTCGGCGGCTTTGTTC \\
\hline \multicolumn{2}{|c|}{ Construction of complementary strain } \\
\hline calR-HP-F & GCGGTCGACAGGAGGAATTCACCATGTTAGAGAAGAAAGATG \\
\hline calR-HP-R & GCGAAGCTTTTATTTTGATGCGACCAC \\
\hline toxR-HP-F & GATTCTAGAAGGAGGAATTCACCATGACTAACATCGGCACCAA \\
\hline toxR-HP-R & GACAAGCTTTTATTTGCAGATGTCTGTTGG \\
\hline \multicolumn{2}{|l|}{ Protein expression } \\
\hline calR-P-F & GCGGGATCCATGTTAGAGAAGAAAGATG \\
\hline calR-P-R & GCGAAGCTTTTATTTTGATGCGACCAC \\
\hline toxR-P-F & AGCGGGATCCATGACTAACATCGGCACCAA \\
\hline toxR-P-R & AGCGAAGCTTTTAAGGATTCACAGCAGAAG \\
\hline \multicolumn{2}{|l|}{ qRT-PCR } \\
\hline calR-RT-F & ATGTAAAAAGAAAACCGTACA \\
\hline calR-RT-R & AACACAGCAGAATGACCGTG \\
\hline toxR-RT-F & TTGTTTGGCGTGAGCAAGG \\
\hline toxR-RT-R & TAGCAGAGGCGTCATTGTTATC \\
\hline $\operatorname{exs} B-\mathrm{RT}-\mathrm{F}$ & ATGAAAAGCAGTAAGTGGGC \\
\hline $\operatorname{exs} B-\mathrm{RT}-\mathrm{R}$ & CTGAGAAGCAACAGTAAGAC \\
\hline vp1687-RT-F & TGCTCACCGTTGCCAAATAG \\
\hline vp1687-RT-R & GCGACGCTTTCATGTATTGC \\
\hline$v p 1667-\mathrm{RT}-\mathrm{R}$ & GGAATGGATTGGAATCGTC \\
\hline$v p 1667-\mathrm{RT}-\mathrm{R}$ & CCACCGTCTTTTATTTTGC \\
\hline 16S rDNA-RT-F & GACACGGTCCAGACTCCTAC \\
\hline 16S rDNA-RT-R & GGTGCTTCTTCTGTCGCTAAC \\
\hline \multicolumn{2}{|l|}{ Primer extension } \\
\hline calR-PE-R & GCAAAATATCGGTACTTCA \\
\hline toxR-PE-R & TTAGTTCTTCTTAGATGGATGATG \\
\hline $\operatorname{exs} B-\mathrm{PE}-\mathrm{R}$ & GTCTTATTATGATTTATTTTTACAC \\
\hline$v p 1687-\mathrm{PE}-\mathrm{R}$ & GGCAACGGTGAGCAAAATC \\
\hline
\end{tabular}

(Continued) 


\begin{tabular}{|c|c|}
\hline Type of analysis and primer & Sequences $\left(5^{\prime}-3^{\prime}\right)$ \\
\hline vp1667-PE-R & GACGATTCCAATCCATTCCG \\
\hline \multicolumn{2}{|l|}{ LacZ fusion } \\
\hline calR-lacZ-F & GCGGTCGACGTTTGTTTGCTCGGATTGTTTG \\
\hline calR-lacZ-R & GCGTCTAGACAAAGTGCTTTCCATACGGTAG \\
\hline toxR-lacZ-F & GCGCGTCGACATCGTTAAGGTATTTGCA \\
\hline toxR-lacZ-R & GCGCGAATTCCGAGCGAATTACTATTTGG \\
\hline exsB-lacZ-F & ATATGTCGACATTGTCCGTCAAATGCAGTTC \\
\hline exsB-lacZ-R & TTTTGAATTC CATATACATTCGCTTGGCTCTG \\
\hline vp1687-lacZ-F & GCGCGTCGACGCATTATTGACGCCAGTATCG \\
\hline vp1687-lacZ-R & GCGCTCTAGAGGCAACGGTGAGCAAAATC \\
\hline vp1667-lacZ-F & GCGGTCGACCAGATTGCTGAATATCGGTG \\
\hline vp1667-lacZ-R & GCGTCTAGA AAGCGATTGAGTGGCGTTG \\
\hline \multicolumn{2}{|l|}{ EMSA } \\
\hline calR-EMSA-F1 & GTTTGTTTGCTCGGATTGTTTG \\
\hline calR-EMSA-R & CAAAGTGCTTTCCATACGGTAG \\
\hline toxR-EMSA-F & ATCGTTAAGGTATTTGCA \\
\hline toxR-EMSA-R & CGAGCGAATTACTATTTGG \\
\hline exs $B$-EMSA-F & ATTGTCCGTCAAATGCAGTTC \\
\hline exs $B$-EMSA-R & CATATACATTCGCTTGGCTCTG \\
\hline$v p 1687-\mathrm{EMSA}-\mathrm{F}$ & GCATTATTGACGCCAGTATCG \\
\hline vp1687-EMSA-R & GGCAACGGTGAGCAAAATC \\
\hline vp1667-EMSA-F & CAGATTGCTGAATATCGGTG \\
\hline vp1667-EMSA-R & AAGCGATTGAGTGGCGTTG \\
\hline \multicolumn{2}{|l|}{ DNase I footprinting } \\
\hline calR-FP-F & CAGATTGCTGAATATCGGTG \\
\hline calR-FP-R & ATTGATAATACTCATTCACTTGC \\
\hline calR-FP-F (M13F) & GTAAAACGACGGCCAGTCCGTTGGTTATTGATAG \\
\hline calR-FP-R (M13R) & CAGGAAACAGCTATGACCCACGGCATTACTTACTG \\
\hline toxR-FP-F (M13F) & GTAAAACGACGGCCAGTTTTCAGGGACGACTTTGTG \\
\hline toxR-FP-R (M13R) & CAGGAAACAGCTATGACCGAGCGAATTACTATTTGG \\
\hline exsB-FP-F (M13F) & GTAAAACGACGGCCAGTGTTTATCAATTTTGGTTGTTAG \\
\hline exsB-FP-R (M13R) & CAGGAAACAGCTATGACCGGCTTATATTTATTCTAC \\
\hline$v p 1687-\mathrm{FP}-\mathrm{F}(\mathrm{M} 13 \mathrm{~F})$ & GTAAAACGACGGCCAGTCACCAGAGTAGGGCATCAC \\
\hline vp1687-FP-R (M13R) & CAGGAAACAGCTATGACCAAGCCAATGAGCGTCAG \\
\hline$v p 1667-\mathrm{FP}-\mathrm{F}(\mathrm{M} 13 \mathrm{~F})$ & GTAAAACGACGGCCAGTCAGATTGCTGAATATCGGTG \\
\hline vp1667-FP-R (M13R) & CAGGAAACAGCTATGACAAGCGATTGAGTGGCGTTG \\
\hline M13F-FAM & GTAAAACGACGGCCAGT \\
\hline M13R-HEX & CAGGAAACAGCTATGAC \\
\hline
\end{tabular}


demonstrated that ToxR inhibits the expression of T3SS1 genes via positive regulation of calR [23]. In addition, the quorum sensing system in $V$. parahaemolyticus appears to activate and repress T3SS1 regulons at low-cell density and high-cell density, respectively [36, 37]. Moreover, the small RNA Spot 42 is involved in regulating the expression of T3SS1 gene $v p 1682$ at post-transcriptional level in $V$. parahaemolyticus, which contributes to cytotoxicity in vivo [38].

The present work demonstrates that $V$. parahaemolyticus ToxR binds to the promoter DNA region from 286 to $257 \mathrm{bp}$ upstream of calR to activate its transcription. CalR occupies the promoter-proximal regions of the three operons (exs $B A D$ $v s c B C D$, vp 1687-1686, and vp1667-1655) in T3SS1 locus to repress their transcription, thereby inhibiting T3SS1dependent cytotoxicity phenotype. Thus, ToxR inhibits T3SS1 expression via direct activation of calR. As a feedback loop, CalR binds to the promoter-proximal regions of toxR and its own gene to inhibit their transcription. Thus, we report an interesting gene regulatory network involving the reciprocal negative regulation of ToxR and CalR, and negative regulation of T3SS1 and calR by CalR in $V$. parahaemolyticus.

The structural organization of each target promoter was reconstructed based on the collection of translation/ transcription start sites, -10 and -35 elements, SD sequences, and ToxR or/and CalR binding sites (Figure 7). The ToxR site for calR is far upstream of the -35 element, and thus the ToxR-dependent calR promoter may belong to class I transcriptional stimulation [39]. The CalR sites for calR and vp1687 overlaps or downstream of the -10 element, the binding of CalR to the calR or $v p 1687$ promoter region would block the entry or elongation of RNA polymerase (RNAP), and a similar mechanism has been observed for Yersinia pestis PhoP [40]. CalR bound to two sites within the upstream region of the tox $R$ and $v p 1667$, and one of the two sites for each gene overlaps or downstream of the transcriptional start site, these two binding sites would form a hairpin in the promoter and thus block the entry or elongation of RNAP. The CalR site for $\operatorname{exs} B$ is located upstream of the -35 element, which is highly unusual for transcriptional repression. However, a similar mechanism has been observed for CalR inhibition of $t d h 2$ in $V$. parahaemolyticus [26], and there would be a potential transcriptional activator for exs $B$ transcription. Overall, $V$. parahaemolyticus $\mathrm{CalR}$ uses a variety of mechanisms to regulate the transcription of its target genes. Nevertheless, whether ToxR have direct regulation on its own gene and T3SS1 genes need to be further characterized.

\section{MATERIALS AND METHODS}

\section{Bacterial strains and plasmids}

The $V$. parahaemolyticus strain RIMD2210633 was used as the wild type (WT) in this study [5]. The calR deletion mutant $(\triangle c a l R)$ was generated as previously described [41, 42]. Briefly, the 405 and 426 bp DNA regions upstream and downstream of $c a l R$ were amplified by PCR using primers calR-A and -B, and calR-C and -D, respectively. After being purified, the amplicons were used as the templates to create an $801 \mathrm{bp}$ deletion construct using primers calR-A and -D, which was subsequently inserted between the PstI and SphI sites of the pDS132 containing the $s a c B$ gene conferring sensitivity to sucrose, and a chloramphenicol resistance gene. After being verified by DNA sequencing, the recombinant vector was transformed into Escherichia coli $\mathrm{S} 17-1(\lambda$ pir), and then transferred into WT by conjugation. The $\Delta$ calR was selected using resistance to $10 \%$ sucrose and sensitivity to $5 \mu \mathrm{g} / \mathrm{ml}$ chloramphenicol, and further verified by PCR. Using the same method, the entire coding region of toxR was deleted from WT genome to create tox $R$ deletion mutant $(\Delta t o x R)$. All the primers used in the present work were listed in Table 1.

To complement the $\Delta c a l R$ or $\Delta$ toxR [35], a PCRgenerated DNA fragment containing the calR or toxR coding region together with an upstream synthetic SD sequence (AGGAGG) was inserted between the $\mathrm{Sal}$ I and Hind III sites of the vector pBAD33 harboring an arabinose $\mathrm{P}_{\mathrm{BAD}}$ promoter and a chloramphenicol resistance gene. After beingverified by DNA sequencing, the recombinant pBAD33-calR or $\mathrm{pBAD} 33$-tox $R$ plasmid was transformed into $\Delta$ calR or $\Delta$ tox $R$, yielding the complemented mutant strain $\Delta$ calR/pBAD33-calR or $\Delta$ toxR/pBAD33-tox $R$. For controls, the empty vector pBAD33 was also transformed into WT and $\Delta$ calR or $\Delta$ toxR to generate $\mathrm{WT} / \mathrm{pBAD} 33$ and $\Delta c a l R / \mathrm{pBAD} 33$ or $\Delta$ toxR/pBAD33.

\section{Bacterial growth conditions}

$V$. parahaemolyticus was cultured in complete HI broth containing 2.5\% Bacto heart infusion (BD Bioscience) at $37{ }^{\circ} \mathrm{C}$ with shaking at $250 \mathrm{rpm}$. The glyceric stock of bacterial cells were inoculated into $5 \mathrm{ml}$ of HI broth and incubated overnight for at least over $14 \mathrm{~h}$. The overnight cell cultures were diluted 1: 50 into $15 \mathrm{ml}$ of fresh HI broth, and grown to reach at $\mathrm{OD}_{600} \approx 1.0$ to 1.2, and then diluted 1:1000 into $15 \mathrm{ml}$ of $\mathrm{HI}$ broth for the third-round growth, and were harvested at an $\mathrm{OD}_{600}$ value of about 1.0 to 1.2 . When required, the culture medium was supplemented with $50 \mu \mathrm{g} / \mathrm{ml}$ gentamicin, $5 \mu \mathrm{g} / \mathrm{ml}$ chloramphenicol, or $0.1 \%$ arabinose.

\section{RNA isolation and quantitative real-time PCR (qRT-PCR)}

Total RNAs were extracted using the TRIzol reagent (Invitrogen). RNA quality and quantity were monitored by agarose gel electrophoresis and spectrophotometry, respectively $[41,42]$. The contaminated genome DNA in the total RNAs was removed by using the Ambion's DNA- 
free $^{\mathrm{TM}} \mathrm{Kit}$. cDNAs were generated by using $3-8 \mu \mathrm{g}$ of RNA and $3 \mu \mathrm{g}$ of random hexamer primers. The SYBR Green qRT-PCR assay was performed and analyzed as previously described [43]. The experiment was performed with at least three independent cultures and RNA preparations.

\section{Primer extension assay}

For the primer extension assay [41, 42], 3-10 $\mu \mathrm{g}$ of total RNAs was annealed with 1 pmol of 5'- ${ }^{32} \mathrm{P}$-labeled reverse oligonucleotide primer to generate cDNAs using a Primer Extension System (Promega) according to the manufacturer's instructions. The same labeled primer was used for sequencing with the AccuPower \& Top DNA Sequencing Kit (Bioneer). The primer extension products and sequencing materials were concentrated and analyzed in an $8 \mathrm{M}$ urea- $6 \%$ polyacrylamide gel electrophoresis, and the results were detected by autoradiography with the Fuji Medical X-ray film.

\section{LacZ fusion and $\beta$-galactosidase assay}

The promoter DNA region of each indicated gene was amplified and cloned into the corresponding restriction endonuclease sites of low-copy-number plasmid pHRP309 that harbors a gentamicin resistance gene and a promoterless lacZ reporter gene [44]. After being verified by DNA sequencing, the recombinant pHRP309 plasmid was transferred into $V$. parahaemolyticus strains. An empty pHRP309 plasmid was also introduced into each strain tested as the negative control. The $V$. parahaemolyticus strains transformed with recombinant or empty pHRP309 plasmids were grown as above to measure the $\beta$-galactosidase activity in cellular extracts using the $\beta$-Galactosidase Enzyme Assay System (Promega) according to the manufacturer's instructions.

\section{Preparation of $6 \times$ His-tagged CalR (His-CalR) and (His-ToxR) proteins}

The entire coding region of calR or the truncated toxR (1-528 bp, a.a.1-176) was amplified, purified, and cloned into pET28a (Novagen). The recombinant plasmid encoding His-CalR or His-ToxR was then transformed

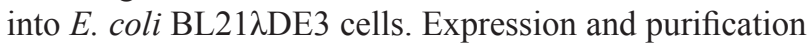
of His-CalR were similar to that of His-OpaR[41], while His-ToxR was the same as that of His-AphA [42]. The purified proteins were concentrated with nickel loaded HiTrap Chelating Sepharose columns (Amersham) and concentrated to a final concentration of about $0.3-0.6 \mathrm{mg} /$ $\mathrm{ml}$. The purified proteins were stored at $-80^{\circ} \mathrm{C}$, and the protein purity was confirmed by SDS-PAGE.

\section{Electrophoretic mobility shift assay (EMSA)}

EMSA was performed with two methods according to the difference in raw material: the radioactive labeled probe and ethidium bromide (EB) stain method [26, 41]. For the EB stain method [26], DNA binding was performed in a $10 \mu \mathrm{l}$ reaction volume containing binding buffer $\left[1 \mathrm{mM} \mathrm{MgCl}_{2}, 0.5 \mathrm{mM}\right.$ EDTA, $0.5 \mathrm{mM}$ DTT, $50 \mathrm{mM} \mathrm{NaCl}, 10 \mathrm{mM}$ Tris- $\mathrm{HCl}$ (pH 7.5) and $10 \mathrm{mg} / \mathrm{ml}$ salmon sperm DNA], 100-200 ng target promoter DNA, and increasing amounts of His-CalR. After incubation at room temperature for $20 \mathrm{~min}$, the products were loaded onto a native $6 \%$ $(\mathrm{w} / \mathrm{v})$ polyacrylamide gel, and electrophoresed in $0.5 \times$ TBE buffer for about $90 \mathrm{~min}$ at $200 \mathrm{~V}$. The gel was examined with a UV transilluminator after staining with the EB dye. For the radioactive labeled probe method [41], the 5' ends of target DNA fragments were labeled using $\left[\gamma-{ }^{32} \mathrm{P}\right]$ ATP and T4 polynucleotide kinase. DNA binding was also performed in a $10 \mu \mathrm{l}$ reaction volume containing binding buffer, radio-labeled DNA, and increasing amounts of His-ToxR. Three controls were included in each EMSA experiment: 1) cold probe as specific DNA competitor (the same promoterproximal DNA region unlabeled), 2) negative probe as non-specific DNA competitor (the unlabeled coding region of the $16 \mathrm{~S}$ rRNA gene), and 3) a non-specific protein competitor (rabbit anti-F1-protein polyclonal antibodies). Radioactive species were detected by autoradiography after exposure to Fuji Medical X-ray film.

\section{DNase I footprinting assay}

DNase I footprinting assays were performed with two different methods: the radioactive labeled probe and DNA sequencing methods. For the radioactive labeled probe method [41, 42], the promoter-proximal DNA region with a single $32 \mathrm{P}$-labeled end was PCR amplified with either sense or antisense end-labeled primers. The PCR products were purified using QiaQuick columns (Qiagen). Increasing amounts of His-ToxR were incubated with the purified and labeled DNA fragments $(2-5 \mathrm{pmol})$ for $30 \mathrm{~min}$ at room temperature. The final reaction volume was $10 \mu \mathrm{l}$ and contained binding buffer used in EMSA. Before DNA digestion, $10 \mu \mathrm{l}$ of $\mathrm{Ca} 2+/ \mathrm{Mg} 2+$ solution (5 $\mathrm{mM} \mathrm{CaCl} 2$ and $10 \mathrm{mM} \mathrm{MgCl} 2$ ) was added, followed by incubation for $1 \mathrm{~min}$ at room temperature. The optimized RQ1 RNase-Free DNase I (Promega) was then added to the reaction mixture, and the mixture was incubated at room temperature for 40-90 s. The reaction was quenched by adding $9 \mu \mathrm{l}$ of stop solution (200 mM NaCl, $30 \mathrm{mM}$ EDTA, and 1\% SDS), followed by incubation for $1 \mathrm{~min}$ at room temperature. The partially digested DNA samples were then extracted with phenol/chloroform, precipitated with ethanol, and analyzed in $6 \%$ polyacrylamide $/ 8 \mathrm{M}$ urea gels. Protected regions were identified by comparison with sequencing ladders. The templates for these sequencing ladders were the same as the DNA fragments for DNase I footprinting. Radioactive species were detected by autoradiography. 
For the DNA sequencing method [26, 28], a DNA fragment of promoter DNA region of each indicated genes was PCR amplified using the primers target-FP-F (M13F) and target-FP-R (M13R) with ExTaq DNA ploymerase. After being purified, the amplicon was used as the template for labeling the probes with different primer pairs: M13FFAM and target gene-FP-R (M13R) for preparation of 6-carboxyfluorescein (FAM)-labeled coding strand, and target gene-FP-F (M13F) and M13R-HEX for preparation of 5'-Hexachlorofluorescein phosphoramidite (HEX)labeled noncoding strand. The PCR products were purified by using the Qiaquick columns (Qiagen) and quantified with a NanoDrop 2000 (Thermo). Approximately $350 \mathrm{ng}$ of the purified, FAM/HEX-labeled DNA fragments were incubated with increasing amounts of His-CalR in a final $10 \mu 1$ reaction volume containing the binding buffer used in EMSA. The DNA digestion procedure was the same as the radioactive labeled probe method. The digested DNA samples were extracted with a Beaver Beads TMPCR Purification Kit (Beaver) according to the manufacturer's instructions, and the sample pellets were dissolved in $15 \mu 1$ modified water (HiDi: water: $600 \mathrm{LIZ}=90: 60: 1$ ). For sequencing, the BigDye ${ }^{\circledR}$ Terminator v3.1 Cycle Sequencing Kits (ABI) was used. The volume of each sequencing reaction was increased to $20 \mu \mathrm{l}$ that contains 10 ng of target promoter region as template, $3.2 \mathrm{pmol}$ of sense or antisense primer as the sequencing primer, and $8 \mu \mathrm{l}$ of BigDye reaction mix (BigDye: $5 \times$ buffer $=1: 3$ ). After pre-denaturation at $96^{\circ} \mathrm{C}$ for $1 \mathrm{~min}, \mathrm{PCR}$ amplification was conducted at 25 cycles of denaturation at $96^{\circ} \mathrm{C}$ for $10 \mathrm{~s}$, annealing at $50^{\circ} \mathrm{C}$ for $5 \mathrm{~s}$ and extension at $60^{\circ} \mathrm{C}$ for $4 \mathrm{~min}$. The sequencing samples were precipitated with the same method as above, and then dissolved in $10 \mu \mathrm{l}$ HiDi and $1 \mu \mathrm{l} 600$ LIZ. The digested DNA fragments were analyzed by using ABI 3500XL DNA Genetic analyzer with GeneMarker software 2.2. The sequencing products were examined with Sequence Scanner software v1.0.

\section{Cell culture and cytotoxicity assay}

HeLa cell was maintained in DMEM (Invitrogen) containing $10 \%$ fetal bovine serum (Invitrogen) at $37^{\circ} \mathrm{C}$ in $5 \% \mathrm{CO}_{2}$. The cytotoxic assays were performed as described previously $[6,35]$. The precultivated bacterial cells were washed and serially ten-fold diluted with the pre-warmed Dulbecco's modified Eagle's medium (DMEM) lacking phenol red for CFU measurement and infection. HeLa cells were infected with $10^{6} \mathrm{CFU}$ of bacteria for $3 \mathrm{~h}$ at a multiplicity of infection (MOI) of 2.5. After infection, the release of lactate dehydrogenase (LDH) into the medium was quantified with a CytoTox96 kit (Promega) according to the manufacturer's instructions.

\section{Experimental replicates and statistical methods}

The LacZ fusion, qRT-PCR and cytotoxicity assays were performed with at least three independent bacterial cultures and the values were expressed as mean \pm standard deviation. Paired Student's $t$-test was used to calculate statistically significant differences, $p<0.01$ was considered to indicate statistical significance. The presented data of primer extension, DNase I footprinting and EMSA assays were done with at least two independent biological replicates.

\section{CONFLICTS OF INTEREST}

The authors declare that no conflicts of interest exists.

\section{FINANCIAL SUPPORT}

This work was supported by the National Natural Science Foundation of China (81601809, 31671290).

\section{REFERENCES}

1. Broberg CA, Calder TJ, Orth K. Vibrio parahaemolyticus cell biology and pathogenicity determinants. Microbes Infect. 2011; 13: 992-1001. doi: 10.1016/j. micinf.2011.06.013.

2. Daniels NA, MacKinnon L, Bishop R, Altekruse S, Ray B, Hammond RM, Thompson S, Wilson S, Bean NH, Griffin PM, Slutsker L. Vibrio parahaemolyticus infections in the United States, 1973-1998. J Infect Dis. 2000; 181: 1661-6. doi: $10.1086 / 315459$.

3. Su YC, Liu C. Vibrio parahaemolyticus: a concern of seafood safety. Food Microbiol. 2007; 24: 549-58. doi: 10.1016/j.fm.2007.01.005.

4. Coburn B, Sekirov I, Finlay BB. Type III secretion systems and disease. Clin Microbiol Rev. 2007; 20: 535-49. doi: 10.1128/CMR.00013-07.

5. Makino K, Oshima K, Kurokawa K, Yokoyama K, Uda T, Tagomori K, Iijima Y, Najima M, Nakano M, Yamashita A, Kubota Y, Kimura S, Yasunaga T, et al. Genome sequence of Vibrio parahaemolyticus: a pathogenic mechanism distinct from that of V. cholerae. Lancet. 2003; 361: 743-9. doi: 10.1016/S0140-6736(03)12659-1.

6. Hiyoshi H, Kodama T, Iida T, Honda T. Contribution of Vibrio parahaemolyticus virulence factors to cytotoxicity, enterotoxicity, and lethality in mice. Infect Immun. 2010; 78: 1772-80. doi: 10.1128/IAI.01051-09.

7. Ono T, Park KS, Ueta M, Iida T, Honda T. Identification of proteins secreted via Vibrio parahaemolyticus type III secretion system 1. Infect Immun. 2006; 74: 1032-42. doi: 10.1128/IAI.74.2.1032-1042.2006.

8. Broberg CA, Zhang L, Gonzalez H, Laskowski-Arce MA, Orth K. A Vibrio effector protein is an inositol phosphatase and disrupts host cell membrane integrity. Science. 2010; 329: 1660-2. doi: 10.1126/science.1192850.

9. Burdette DL, Yarbrough ML, Orvedahl A, Gilpin CJ, Orth K. Vibrio parahaemolyticus orchestrates a multifaceted host 
cell infection by induction of autophagy, cell rounding, and then cell lysis. Proc Natl Acad Sci U S A. 2008; 105: $12497-$ 502. doi: 10.1073/pnas.0802773105.

10. Kodama T, Rokuda M, Park KS, Cantarelli VV, Matsuda $\mathrm{S}$, Iida T, Honda T. Identification and characterization of VopT, a novel ADP-ribosyltransferase effector protein secreted via the Vibrio parahaemolyticus type III secretion system 2. Cell Microbiol. 2007; 9: 2598-609. doi: 10.1111/j.1462-5822.2007.00980.x.

11. Burdette DL, Seemann J, Orth K. Vibrio VopQ induces PI3-kinase-independent autophagy and antagonizes phagocytosis. Mol Microbiol. 2009; 73: 639-49. doi: 10.1111/j.1365-2958.2009.06798.x.

12. Shimohata $T$, Nakano M, Lian X, Shigeyama T, Iba H, Hamamoto A, Yoshida M, Harada N, Yamamoto H, Yamato M, Mawatari K, Tamaki T, Nakaya Y, et al. Vibrio parahaemolyticus infection induces modulation of IL-8 secretion through dual pathway via VP1680 in Caco-2 cells. J Infect Dis. 2011; 203: 537-44. doi: 10.1093/infdis/jiq070.

13. Matlawska-Wasowska K, Finn R, Mustel A, O’Byrne CP, Baird AW, Coffey ET, Boyd A. The Vibrio parahaemolyticus Type III Secretion Systems manipulate host cell MAPK for critical steps in pathogenesis. BMC Microbiol. 2010; 10: 329. doi: 10.1186/1471-2180-10-329.

14. Bhattacharjee RN, Park KS, Chen X, Iida T, Honda T, Takeuchi O, Akira S. Translocation of VP1686 upregulates RhoB and accelerates phagocytic activity of macrophage through actin remodeling. J Microbiol Biotechnol. 2008; 18: 171-5.

15. Liverman AD, Cheng HC, Trosky JE, Leung DW, Yarbrough ML, Burdette DL, Rosen MK, Orth K. Arp2/3independent assembly of actin by Vibrio type III effector VopL. Proc Natl Acad Sci U S A. 2007; 104: 17117-22. doi: 10.1073/pnas.0703196104.

16. Higgins DE, DiRita VJ. Transcriptional control of toxT, a regulatory gene in the ToxR regulon of Vibrio cholerae. Mol Microbiol. 1994; 14: 17-29.

17. DiRita VJ, Parsot C, Jander G, Mekalanos JJ. Regulatory cascade controls virulence in Vibrio cholerae. Proc Natl Acad Sci U S A. 1991; 88: 5403-7.

18. Hung DT, Mekalanos JJ. Bile acids induce cholera toxin expression in Vibrio cholerae in a ToxT-independent manner. Proc Natl Acad Sci U S A. 2005; 102: 3028-33. doi: 10.1073/pnas.0409559102.

19. Provenzano D, Klose KE. Altered expression of the ToxRregulated porins $\mathrm{OmpU}$ and $\mathrm{OmpT}$ diminishes Vibrio cholerae bile resistance, virulence factor expression, and intestinal colonization. Proc Natl Acad Sci U S A. 2000; 97: 10220-4. doi: 10.1073/pnas.170219997.

20. Provenzano D, Lauriano CM, Klose KE. Characterization of the role of the ToxR-modulated outer membrane porins OmpU and OmpT in Vibrio cholerae virulence. J Bacteriol. 2001; 183: 3652-62. doi: 10.1128/ JB.183.12.3652-3662.2001.
21. Kazi MI, Conrado AR, Mey AR, Payne SM, Davies BW. ToxR antagonizes H-NS regulation of horizontally acquired genes to drive host colonization. PLoS Pathog. 2016; 12: e1005570. doi: 10.1371/journal.ppat.1005570.

22. Lin Z, Kumagai K, Baba K, Mekalanos JJ, Nishibuchi M. Vibrio parahaemolyticus has a homolog of the Vibrio cholerae toxRS operon that mediates environmentally induced regulation of the thermostable direct hemolysin gene. J Bacteriol. 1993; 175: 3844-55.

23. Whitaker WB, Parent MA, Boyd A, Richards GP, Boyd EF. The Vibrio parahaemolyticus ToxRS regulator is required for stress tolerance and colonization in a novel orogastric streptomycin-induced adult murine model. Infect Immun. 2012; 80: 1834-45. doi: 10.1128/IAI.06284-11.

24. Hubbard TP, Chao MC, Abel S, Blondel CJ, Abel Zur Wiesch P, Zhou X, Davis BM, Waldor MK. Genetic analysis of Vibrio parahaemolyticus intestinal colonization. Proc Natl Acad Sci U S A. 2016; 113: 6283-8. doi: 10.1073/ pnas. 1601718113.

25. Gode-Potratz CJ, Chodur DM, McCarter LL. Calcium and iron regulate swarming and type III secretion in Vibrio parahaemolyticus. J Bacteriol. 2010; 192: 6025-38. doi: 10.1128/JB.00654-10.

26. Zhang Y, Gao H, Zhang L, Yin Z, Huang X, Zhou D, Yang H, Yang W, Wang L. Vibrio parahaemolyticus CalR down regulates the thermostable direct hemolysin (TDH) gene transcription and thereby inhibits hemolytic activity. Gene. 2017; 613: 39-44. doi: 10.1016/j.gene.2017.03.001.

27. Zhang L, Osei-Adjei G, Zhang Y, Gao H, Yang W, Zhou D, Huang X, Yang H. CalR is required for the expression of T6SS2 and the adhesion of Vibrio parahaemolyticus to HeLa cells. Arch Microbiol. 2017; 199: 931-8. doi: 10.1007/s00203-017-1361-6.

28. Gao H, Zhang L, Osei-Adjei G, Yang W, Zhou D, Huang X, Yang H, Yin Z, Zhang Y. Transcriptional regulation of cpsQmfpABC and mfpABC by CalR in Vibrio parahaemolyticus. Microbiologyopen. 2017. doi: 10.1002/mbo3.470.

29. Yang L, Zhou D, Liu X, Han H, Zhan L, Guo Z, Zhang L, Qin C, Wong HC, Yang R. Cold-induced gene expression profiles of Vibrio parahaemolyticus: a time-course analysis. FEMS Microbiol Lett. 2009; 291: 50-8. doi: 10.1111/j.1574-6968.2008.01434.x.

30. Yang L, Zhan L, Han H, Gao H, Guo Z, Qin C, Yang R, Liu X, Zhou D. The low-salt stimulon in Vibrio parahaemolyticus. Int J Food Microbiol. 2010; 137: 49-54. doi: 10.1016/j.ijfoodmicro.2009.11.006.

31. Yahr TL, Wolfgang MC. Transcriptional regulation of the Pseudomonas aeruginosa type III secretion system. Mol Microbiol. 2006; 62: 631-40. doi: 10.1111/j.1365-2958.2006.05412.x.

32. Zhou X, Konkel ME, Call DR. Regulation of type III secretion system 1 gene expression in Vibrio parahaemolyticus is dependent on interactions between 
ExsA, ExsC, and ExsD. Virulence. 2010; 1: 260-72. doi: 10.4161/viru.1.4.12318.

33. Erwin DP, Nydam SD, Call DR. Vibrio parahaemolyticus ExsE is requisite for initial adhesion and subsequent type III secretion system 1-dependent autophagy in HeLa cells. Microbiology. 2012; 158: 2303-14. doi: 10.1099/ mic.0.059931-0.

34. Zhang Y, Osei-Adjei G, Ni B, Fang H, Zhang L, Zhao X, Huang X, Yang H, Yang W, Sun F. Transcription of exsD is repressed directly by H-NS in Vibrio parahaemolyticus. Microb Pathog. 2016; 97: 221-5. doi: 10.1016/j. micpath.2016.06.003.

35. Sun F, Zhang Y, Qiu Y, Yang H, Yang W, Yin Z, Wang J, Yang R, Xia P, Zhou D. H-NS is a repressor of major virulence gene loci in Vibrio parahaemolyticus. Front Microbiol. 2014; 5: 675. doi: 10.3389/fmicb.2014.00675.

36. Wang L, Ling Y, Jiang H, Qiu Y, Qiu J, Chen H, Yang R, Zhou D. AphA is required for biofilm formation, motility, and virulence in pandemic Vibrio parahaemolyticus. Int $\mathrm{J}$ Food Microbiol. 2013; 160: 245-51.

37. Gode-Potratz CJ, McCarter LL. Quorum sensing and silencing in Vibrio parahaemolyticus. J Bacteriol. 2011; 193: 4224-37. doi: 10.1128/JB.00432-11.

38. Tanabe T, Miyamoto K, Tsujibo H, Yamamoto S, Funahashi T. The small RNA Spot 42 regulates the expression of the type III secretion system 1 (T3SS1) chaperone protein VP1682 in Vibrio parahaemolyticus. FEMS Microbiol Lett. 2015; 362. doi: 10.1093/femsle/fnv173.
39. Ishihama A. Functional modulation of Escherichia coli RNA polymerase. Annu Rev Microbiol. 2000; 54: 499-518. doi: 10.1146/annurev.micro.54.1.499.

40. Zhang Y, Wang L, Fang N, Qu S, Tan Y, Guo Z, Qiu J, Zhou D, Yang R. Reciprocal regulation of $\mathrm{pH} 6$ antigen gene loci by PhoP and RovA in Yersinia pestis biovar Microtus. Future Microbiol. 2013; 8: 271-80. doi: 10.2217/ fmb.12.146.

41. Zhang Y, Qiu Y, Tan Y, Guo Z, Yang R, Zhou D. Transcriptional regulation of opaR, qrr2-4 and aphA by the master quorum-sensing regulator OpaR in Vibrio parahaemolyticus. PLoS One. 2012; 7: e34622. doi: 10.1371/journal.pone.0034622.

42. Sun F, Zhang Y, Wang L, Yan X, Tan Y, Guo Z, Qiu J, Yang $\mathrm{R}$, Xia P, Zhou D. Molecular characterization of direct target genes and cis-acting consensus recognized by quorumsensing regulator AphA in Vibrio parahaemolyticus. PLoS One. 2012; 7: e44210. doi: 10.1371/journal.pone.0044210.

43. Gao H, Zhang Y, Yang L, Liu X, Guo Z, Tan Y, Han Y, Huang X, Zhou D, Yang R. Regulatory effects of cAMP receptor protein (CRP) on porin genes and its own gene in Yersinia pestis. BMC Microbiol. 2011; 11: 40. doi: 10.1186/1471-2180-11-40.

44. Parales RE, Harwood CS. Construction and use of a new broad-host-range lacZ transcriptional fusion vector, pHRP309, for gram- bacteria. Gene. 1993; 133: 23-30. 